\title{
Monitoring mitochondrial translation by pulse SILAC
}

Koshi Imami' ${ }^{1,2}$, Matthias Selbach ${ }^{3}$, Yasushi Ishihama ${ }^{1,4 *}$

${ }^{1}$ Graduate School of Pharmaceutical Sciences, Kyoto University, Kyoto 606-8501, Japan

${ }^{2}$ PRESTO, Japan Science and Technology Agency (JST), 5-3 Yonban-cho, Chiyoda-ku, Tokyo, 102-0075, Japan

${ }^{3}$ Max Delbrück Center for Molecular Medicine in the Helmholtz Association (MDC), 13125 Berlin, Germany

${ }^{4}$ Laboratory of Clinical and Analytical Chemistry, National Institute of Biomedical Innovation, Health and Nutrition, Ibaraki, Osaka, 567-0085, Japan

${ }^{*}$ Corresponding authors:

Koshi Imami (Lead contact)

Department of Molecular and Cellular BioAnalysis, Graduate School of Pharmaceutical Sciences, Kyoto University, Kyoto 606-8501, Japan

Tel.: +81-75-753-4565

Fax.: +81-75-753-4601

email: imami.koshi.3z@kyoto-u.ac.jp

Yasushi Ishihama

Department of Molecular and Cellular BioAnalysis, Graduate School of Pharmaceutical Sciences, Kyoto University, Kyoto 606-8501, Japan

Tel.: +81-75-753-4555

Fax.: +81-75-753-4601

email: yishiham@pharm.kyoto-u.ac.jp 


\section{Summary}

Mitochondrial ribosomes are specialized to translate the 13 membrane proteins encoded in the mitochondrial genome, but it is challenging to quantify mitochondrial translation products due to their hydrophobic nature. Here, we introduce a proteomic method that combines biochemical isolation of mitochondria with pulse stable isotope labeling by amino acids in cell culture (pSILAC). Our method provides the highest protein coverage (quantifying 12 out of the 13 innermembrane proteins; average 2-fold improvement over previous studies) with the shortest measurement time. We applied this method to uncover the global picture of (post)translational regulation of both mitochondrial- and nuclear-encoded proteins involved in the assembly of protein complexes that mediate oxidative phosphorylation (OXPHOS). The results allow us to infer the assembly order of complex components and/or partners, as exemplified by complex III. This method should be applicable to study mitochondrial translation programs in many contexts, including oxidative stress and mitochondrial disease.

\section{Keywords}

Proteomics, pulse SILAC, Translation, Mitochondria, OXPHOS, Protein complex 


\section{Introduction}

In eukaryotes, both cytosolic and mitochondrial ribosomes (mitoribosomes) play a central role in protein synthesis. Cytosolic ribosomes produce constituents of the cellular proteome encoded in the nuclear genome, while mitoribosomes are specialized to translate the 13 membrane proteins encoded in the mitochondrial genome. These translational products are some of the subunits of oxidative phosphorylation (OXPHOS) complexes that are essential for energy generation in cells. Cytosolic and mitochondrial ribosomes coordinate their translation to enable the proper assembly of OXPHOS complexes on the inner mitochondrial membrane (Couvillion et al., 2016; Dennerlein et al., 2017; Priesnitz and Becker, 2018; Richter-Dennerlein et al., 2016; Tang et al., 2020; Topf et al., 2019). Thus, co-regulation of the mitochondrial and cytosolic translation programs is essential for maintaining mitochondrial proteostasis, i.e., to prevent accumulation of unwanted and potentially harmful assembly intermediates (Isaac et al., 2018). Moreover, many disease-associated mitochondrial mutations are known to impair the mitochondrial translation machinery (Scharfe et al., 2009; Webb et al., 2020), suggesting that dysregulation of mitochondrial translation leads to disease.

Despite the importance of the mitochondrial translation system, a simple and robust method to monitor the mitochondrial translation products is lacking. A classical approach is pulse labeling of mitochondrial translation products with radiolabeled amino acids, such as $\left[{ }^{35}\right.$ S $]$ methionine and $\left[{ }^{35}\right.$ S]cysteine (Chomyn, 1996; Lazarou et al., 2007), but the use of radioactive materials and the low resolution of SDS-PAGE gel-based separation of the products limits the usability of this method. Recently, mass spectrometry (MS)-based proteomic approaches have been developed to monitor protein synthesis (Iwasaki and Ingolia, 2017). Quantitative non-canonical amino acid tagging (QuaNCAT) (Eichelbaum et al., 2012; Howden et al., 2013; Schanzenbächer et al., 2016) relies on pulse labeling of newly synthesized proteins with a methionine analog, azidohomoalanine (AHA) (Dieterich et al., 2006), allowing for selective enrichment of the tagged protein pool through click-chemistry as well as MS-based profiling of the tagged proteins. Nascent chain proteomics using puromycin or its analogs enables isolation and identification of nascent polypeptide chains that are being elongated by the ribosomes (Aviner et al., 2013; Forester et al., 2018; Hünten et al., 2015; Tong et al., 2020; Uchiyama et al., 2020). However, these methods require a considerable number of cells (typically $>10^{7}$ cells), involve multiple steps to purify newly synthesized proteins via affinity purification and/or require the isolation of ribosome complexes through density gradient ultracentrifugation. 
In contrast, pulse stable isotope labeling of amino acids in cell culture (pSILAC) is a simple technique for a global analysis of cellular protein translation (Schwanhäusser et al., 2009; Selbach et al., 2008; Ebner and Selbach, 2014; Hünten et al., 2015; Imami et al., 2018; Klann et al., 2020). pSILAC involves metabolic pulse labeling of newly synthesized proteins with either heavy or medium-heavy amino acids for two cell populations of interest. The newly synthesized (labeled) proteins can be distinguished from pre-existing (non-labeled) proteins by means of MS. The heavy to medium-heavy ratios in the MS spectra reflect the differences in protein production between the two conditions. Of note, dynamic SILAC approach (Doherty et al., 2009), a variant of SILAC to measure protein turnover, was also recently used to study turnover rates of mitochondrial proteins in yeast (Saladi et al., 2020) and in human (Bogenhagen and Haley, 2020). Compared to the methods described above, pSILAC does not require many cells (from one to three orders of magnitude fewer) and the downstream experimental process is simply a conventional proteomic workflow. Thus, pSILAC would be a powerful approach to monitor the mitochondrial translation.

One of the major challenges in the analysis of mitochondrial translation is that mass spectrometric identification and quantification of the 13 mitochondrial membrane proteins is hampered by low protein coverage (Figure 1) due to the hydrophobic nature and relatively low abundance of these proteins. To overcome this problem, here, we present a method to comprehensively monitor protein synthesis by mitoribosomes that combines biochemical isolation of mitochondria with PSILAC. Our method offers the highest protein coverage (quantifying 12 out of the 13 membrane proteins; average 2-fold improvement over previous studies) and the shortest MS measurement time among current methods. To demonstrate the utility of this method, we applied it to examine the global translational regulation of the mitochondrial- and nuclear-encoded protein components of the complexes that mediate oxidative phosphorylation (OXPHOS).

\section{Results and Discussion}

\section{Biochemical optimization for comprehensive analysis of the mitochondrial translational products}

While proteomic technologies to quantify protein synthesis have been developed, comprehensive analysis of the mitochondrial translation products (MT-proteins) is challenging (Figure 1). To address this issue, we first examined whether the quantitation of MT-proteins 
could be improved by combining isolation of mitochondria with the application of combinations of proteases. Chymotrypsin cleaves on the C-terminal hydrophobic essential amino acids (phenylalanine, tryptophan, and tyrosine) and thus might be suitable for digesting membrane proteins and for pSILAC. We adapted a protocol for mitochondrial isolation according to (Frezza et al., 2007), as it is relatively simple (not requiring ultracentrifugation) and quick (approximately $40 \mathrm{~min}$ ) (see Methods). Mitochondria pellets isolated from HEK293T cells were lysed and protein digestion was performed with 1) chymotrypsin, 2) chymotrypsin-lysC, 3) lysC-trypsin or 4) chymotrypsin-trypsin. In parallel, total cell lysates were digested in the same way as a control. Two biological replicates were analyzed, and a complete list of proteins identified is provided in Table S1. We confirmed that mitochondrial proteins were highly enriched in the isolated mitochondrial fractions, as judged by gene ontology enrichment analysis (Figure 2A).

Figures $2 \mathrm{~B}$ and $2 \mathrm{C}$ show the coverage of MT-proteins at the protein- and peptide-levels, respectively. Isolation of mitochondria significantly enhanced the identification of MT-proteins, as compared to the total cell lysate (Figure 2B). In every digestion protocol, 12 out of the 13 MT proteins were identified in the mitochondrial fraction, while on average only 9 proteins were identified in the total lysates. Further, the use of chymotrypsin alone or in combination with lys $\mathrm{C}$ or trypsin slightly increased the number of identified peptides compared to lys C-trypsin digestion (Figure 2C). Importantly, it was possible to identify and quantify all 13 MT-proteins by utilizing a combination of two digestion protocols (e.g., lysC-trypsin and chymotrypsin) after mitochondria isolation. Any one of the tested digestion protocols alone missed one MT-protein because cleavages were limited, resulting in no MS-identifiable peptides of a reasonable length. Given that all of the digestion protocols allowed identification of 12 MT-proteins (Figure 2B), we decided to focus on lysC-trypsin digestion of isolated mitochondria for further analysis, as it produces tryptic peptides that are quantifiable in standard pSILAC (Selbach et al., 2008) without special modifications to the culture medium.

\section{pSILAC approach to monitor mitochondrial translation}

Having established the biochemical condition, we next performed pSILAC experiments. Chloramphenicol (CAP) binds to the A-site crevice on bacterial and mitochondrial ribosomes (Jardetzky, 1963), and thereby inhibits mitochondrial, but not cytosolic translation (Figure 3A). Using CAP, we sought to assess how inhibition of mitochondrial translation impacts on the 
synthesis of both MT-proteins and other nuclear-encoded OXPHOS complex proteins by applying pSILAC methodology.

HEK293T cells cultivated in light medium were switched to medium-heavy or heavy medium. The cells were pulse-labeled with medium-heavy or heavy amino acids in the presence of CAP or DMSO (Figure 3A), and label-swap experiments were performed in biological duplicate. The digested peptides were fractionated into 7 fractions using an SCX StageTip (Adachi et al., 2016), and individual fractions were analyzed by LC/MS/MS (total $14 \mathrm{hr}$ measurement time).

In total, we identified 4,193 proteins, of which 3,501 proteins were quantified in all samples and were used for further analysis (see Table S2 for a complete list of proteins). As expected, our method successfully quantified 12 out of the 13 MT-proteins (Figure 3B). Typical MS spectra for MT-CO2 are shown in Figure 3C. The translation of the MT-proteins was inhibited by CAP treatment (Figure 3B and $3 \mathrm{C}$ ), which demonstrates that our approach is indeed able to capture changes in mitochondrial translation. Together, the developed proteomic method provides nearcomprehensive (92\% coverage) quantification of MT-proteins, representing an improvement of about 2-fold in protein coverage in comparison to previous reports (Figure 1).

\section{Relationship between mitochondrial translation and assembly of OXPHOS complexes}

In addition to MT-proteins, our mitochondria-focused approach yielded good coverage of the OXPHOS complex subunits, including the nuclear-encoded proteins - 41/45 proteins (91\%), 4/4 proteins (100\%), 9/11 proteins (82\%), 14/21 proteins (67\%), and 13/17 proteins (76\%) from complexes I-V, respectively. Therefore, these data can be used to assess how the nuclearencoded subunits are (post-)translationally regulated in concert with the inhibition of mitochondrial translation. We found significant attenuation of the production of some nuclearencoded subunits (e.g., COX8A and COX7C in complex IV) (Figures 4A), though translation of most of the nuclear-encoded OXPHOS subunits remained unchanged.

To better understand this regulation of some nuclear-encoded subunits, we focused on complex I (NADH ubiquinone oxidoreductase), complex IV (cytochrome c oxidase) and complex V (ATP synthase) whose assembly subunits and pathways are well characterized in human (GuerreroCastillo et al., 2017; He et al., 2018; Signes and Fernandez-Vizarra, 2018; Stroud et al., 2016; Vidoni et al., 2017). Complex $\mathrm{I}$ is composed of the seven modules that are assembled individually (Figure 4B). Our pSILAC data indicates that the nascent nuclear-encoded subunits 
residing in the same structural module of the mitochondria-encoded subunits (ND1, ND2, ND4 and ND5-modules) were downregulated (Figure 4B), while the nuclear-encoded subunits (N module, $Q$ module and NDUFAB1) were rather stable. Likewise, we found that the nuclearencoded subunits affected by CAP were direct assembly partners of the MT-proteins of complex IV (MT-CO1, MT-CO2 and MT-CO3) (Figure 4C). In contrast, we observed no significant translational regulation of the early assembly subunits (COX4 and COX5A) that do not form a subcomplex with the MT-proteins. Similar results were obtained for complex V; MT-ATP6 and MT-ATP8 are involved in the late step of the complex assembly, and the nuclear-encoded subunits corresponding to the early and intermediate subcomplexes were not translationally regulated (Figure 4D).

These results support the notion that orphan nuclear-encoded subunits are subject to (post)translational degradation (Isaac et al., 2018; McShane et al., 2016; Taggart et al., 2020) or pausing translation of them (Richter-Dennerlein et al., 2016) to prevent accumulation of unwanted assembly intermediates. This is interesting because our data can throw light on the assembly orders of complex components and/or partners based on quantitative information, analogous to genetic studies in which impacts of the loss of each subunit on the stability of other subunits were investigated (Protasoni et al., 2020; Stroud et al., 2016; Vidoni et al., 2017). To test this idea, we sought to infer the intermediate steps of complex III (cytochrome bc1 complex) assembly, because so far only the first and last steps of its assembly are well understood in human (Fernández-Vizarra and Zeviani, 2015; Signes and Fernandez-Vizarra, 2018). The assembly model of the complex III from yeast (Gruschke et al., 2011, 2012; Hildenbeutel et al., 2014) is shown in Figure 4E (top). In accordance with the initial step, inhibiting the translation of MT-CYB led to (post-)translational repression of its partner subunits, UQCRQ and UQCRB (Figure 4E). Besides, consistent with the last step of the assembly in which the Rieske Fe-S protein, UQCRFS1, waits for and joins pre-complex III assembly (Signes and Fernandez-Vizarra, 2018), orphan UQCRFS1 was (post-)translationally down-regulated (Figure 4E). Intriguingly, in contrast to the yeast model, our results imply that UQCRC1 and UQCRC2 may be incorporated into the early-assembled complex of MT-CYB, UQCRQ and UQCRB during the initial/intermediate steps, as the abundances of the newly synthesized UQCRC1 and UQCRC2 depended on the translation of MT-CYB (Figure 4E). By contrast, other subunits (CYC1, UQCRH and UQCR10) appear to be independent of the presence of the early assembled complex, indicating that these subunits might form distinct module(s). 
In summary, we observed that inhibition of mitochondrial translation induced (post-)translational regulations of the nuclear-encoded proteins of OXPHOS complexes (Figure 4A), and this regulation is the most likely to be maintained at the structural module level; orphan nascent nuclear-encoded proteins can be degraded in concert with the presence of their partner MTproteins in the same structural module. This corroborates previous genetic studies showing that loss of each subunit affects the stability of other subunits residing in the same structural module of complex I and IV (Protasoni et al., 2020; Stroud et al., 2016; Vidoni et al., 2017). Specifically, this study provides insight into the assembly of complex III during the intermediate step (Figure 4E) which has been less appreciated than the initial and late steps. Collectively, our results indicate that OXPHOS biogenesis (complexes I, III and IV) appear to be regulated in a coordinated way in humans as observed in yeast (Couvillion et al., 2016). To our knowledge, this is the first study that showed near-comprehensive coverage of nascent MT-proteins translated by mitoribosomes and that provided the global view of OXPHOS complex assembly on the basis of (post-)translational regulation of mitochondrial- and nuclear-encoded proteins. We believe that further studies using this methodology will accelerate our understanding of mitochondrial translation programs in many contexts, such as oxidative stress and mitochondrial disease.

\section{Experimental procedures}

The detailed description of the experiments regarding cell culture, pulse SILAC labeling mitochondria isolation, digestion, and LC/MS/MS analysis can be found in the Supplemental Information.

\section{Acknowledgements}

We are very grateful to Erik McShane (Harvard Medical School) for critical reading of the manuscript. We thank the members of the Department of Molecular \& Cellular BioAnalysis and the Department of Proteomics and Drug Discovery for fruitful discussion. KI thanks the Samuro Kakiuchi Memorial Research Award for Young Scientists for supporting this study. This work was supported by JSPS Grant-in-Aid for Scientific Research (Grant Numbers JP18K14674, JP2OH03241, JP2OH04844 to KI and 17H05667 to YI), JST PRESTO (JPMJPR18H2), the Takeda Science Foundation to KI, JST Strategic Basic Research Program CREST (18070870), 
and AMED Advanced Research and Development Programs for Medical Innovation CREST (18068699) to $\mathrm{YI}$.

\section{Author contributions}

Conceptualization \& Methodology, K.I.; Investigation, K.I, M.S. and Y.I.; Formal Analysis, K.I. M.S and Y.I; Resources, K.I, M.S. and Y.I; Writing - Original Draft, K.I; Writing - Review \& Editing, M.S. and Y.I., Visualization, K.I; Supervision, K.I. M.S. and Y.I.; Funding Acquisition, K.I. and Y.I.

\section{Declaration of interests}

The authors declare no competing financial interest.

\section{Supporting Information}

Table S1: A list of proteins identified in mitochondria fraction and total lysate (related to Figure 2)

Table S2: A list of proteins quantified in pSILAC experiments (related to Figures 3 and 4)

\section{Figure legends}

Figure 1: Comparison of our method with previous studies.

The coverage of the MT-proteins (left axis; red color) and the total LC/MS/MS measurement time (right axis; grey color) are shown. Methods and cell types are shown in parentheses. The present study gave the highest coverage of MT-proteins (12 proteins) with the shortest measurement time (14 hr).

\section{Figure 2: Optimization of biochemical conditions.}

(A) The top3 GO terms enriched in the mitochondrial fraction (top: light green) and total cell lysate (bottom: grey). (B) Coverage of the MT-proteins obtained under 8 different conditions. The bars show the average number of identified proteins from two independent experiments (filled-circle). (C) A heatmap showing the number of identified peptides per MT-protein.

\section{Figure 3: pSILAC experiments.}


(A) Experimental scheme of pSILAC. HEK293T cells were pulsed-labeled with medium-heavy or heavy amino acids in the presence of CAP or DMSO. Label-swap experiments were performed. (B) A volcano plot showing log2 fold-change (CAP/DMSO control) and -log10 p-value. The MTproteins are indicated by light green filled circles. (C) MS spectrum of an MT-CO2 peptide (VVLPIEAPIR, +2), as an example.

Figure 4: (Post-)translational control and OXPHOS complex assembly.

(A) Box plots showing log2 fold-change (CAP/Control) of nuclear- ("nuc") and mitochondrialencoded proteins ("mito") in individual OXPHOS complexes. (B) Positions of subunits in complex I (NADH ubiquinone oxidoreductase) based on humans (Zhu et al., 2016) (right) and boxplots showing log2 fold-change (CAP/Control) for "nuc" and "mito" proteins. (C) An assembly model for complex IV (or cytochrome c oxidase) based on humans (Vidoni et al., 2017). Quantitative values (i.e., log2 FCs (CAP/Control)) are indicated by color. (D) An assembly model for complex $V$ (or ATP synthase) based on humans (He et al., 2018). (E) A reported assembly model for complex III (cytochrome bc1 complex) based on yeast (Fernández-Vizarra and Zeviani, 2015) (top) and exemplary MS spectra for the nuclear-encoded proteins (UQCRQ, CYC1 and UQCRFS1) derived peptides (bottom).

\section{References}

Adachi, J., Hashiguchi, K., Nagano, M., Sato, M., Sato, A., Fukamizu, K., Ishihama, Y., and Tomonaga, T. (2016). Improved Proteome and Phosphoproteome Analysis on a Cation Exchanger by a Combined Acid and Salt Gradient. Anal. Chem. 88, 7899-7903.

Aviner, R., Geiger, T., and Elroy-Stein, O. (2013). Novel proteomic approach (PUNCH-P) reveals cell cycle-specific fluctuations in mRNA translation. Genes Dev. 27, 1834-1844.

Bogenhagen, D.F., and Haley, J.D. (2020). Pulse-chase SILAC-based analyses reveal selective oversynthesis and rapid turnover of mitochondrial protein components of respiratory complexes. J. Biol. Chem. 295, 2544-2554.

Chomyn, A. (1996). In vivo labeling and analysis of human mitochondrial translation products. Methods Enzymol. 264, 197-211.

Couvillion, M.T., Soto, I.C., Shipkovenska, G., and Churchman, L.S. (2016). Synchronized mitochondrial and cytosolic translation programs. Nature 533, 499-503.

Cox, J., and Mann, M. (2008). MaxQuant enables high peptide identification rates, individualized p.p.b.-range mass accuracies and proteome-wide protein quantification. Nat. Biotechnol. 26, 
1367-1372.

Cox, J., Neuhauser, N., Michalski, A., Scheltema, R.A., Olsen, J.V., and Mann, M. (2011). Andromeda: a peptide search engine integrated into the MaxQuant environment. J. Proteome Res. 10, 1794-1805.

Dennerlein, S., Wang, C., and Rehling, P. (2017). Plasticity of Mitochondrial Translation. Trends Cell Biol. 27, 712-721.

Dieterich, D.C., Link, A.J., Graumann, J., Tirrell, D.A., and Schuman, E.M. (2006). Selective identification of newly synthesized proteins in mammalian cells using bioorthogonal noncanonical amino acid tagging (BONCAT). Proc. Natl. Acad. Sci. U. S. A. 103, 9482-9487.

Doherty, M.K., Hammond, D.E., Clague, M.J., Gaskell, S.J., and Beynon, R.J. (2009). Turnover of the human proteome: determination of protein intracellular stability by dynamic SILAC. J. Proteome Res. 8, 104-112.

Ebner, O.A., and Selbach, M. (2014). Quantitative Proteomic Analysis of Gene Regulation by miR-34a and miR-34c. PLoS One 9, e92166.

Eichelbaum, K., Winter, M., Berriel Diaz, M., Herzig, S., and Krijgsveld, J. (2012). Selective enrichment of newly synthesized proteins for quantitative secretome analysis. Nat. Biotechnol. 30, 984-990.

Fernández-Vizarra, E., and Zeviani, M. (2015). Nuclear gene mutations as the cause of mitochondrial complex III deficiency. Front. Genet. 6, 134.

Forester, C.M., Zhao, Q., Phillips, N.J., Urisman, A., Chalkley, R.J., Oses-Prieto, J.A., Zhang, L., Ruggero, D., and Burlingame, A.L. (2018). Revealing nascent proteomics in signaling pathways and cell differentiation. Proc. Natl. Acad. Sci. U. S. A. 115, 2353-2358.

Frezza, C., Cipolat, S., and Scorrano, L. (2007). Organelle isolation: functional mitochondria from mouse liver, muscle and cultured fibroblasts. Nat. Protoc. 2, 287-295.

Gruschke, S., Kehrein, K., Römpler, K., Gröne, K., Israel, L., Imhof, A., Herrmann, J.M., and Ott, M. (2011). Cbp3-Cbp6 interacts with the yeast mitochondrial ribosomal tunnel exit and promotes cytochrome b synthesis and assembly. J. Cell Biol. 193, 1101-1114.

Gruschke, S., Römpler, K., Hildenbeutel, M., Kehrein, K., Kühl, I., Bonnefoy, N., and Ott, M. (2012). The Cbp3-Cbp6 complex coordinates cytochrome b synthesis with bc1 complex assembly in yeast mitochondria. J. Cell Biol. 199, 137-150.

Guerrero-Castillo, S., Baertling, F., Kownatzki, D., Wessels, H.J., Arnold, S., Brandt, U., and Nijtmans, L. (2017). The Assembly Pathway of Mitochondrial Respiratory Chain Complex I. Cell Metab. 25, 128-139.

He, J., Ford, H.C., Carroll, J., Douglas, C., Gonzales, E., Ding, S., Fearnley, I.M., and Walker, J.E. (2018). Assembly of the membrane domain of ATP synthase in human mitochondria. Proc. Natl. Acad. Sci. U. S. A. 115, 2988-2993.

Hildenbeutel, M., Hegg, E.L., Stephan, K., Gruschke, S., Meunier, B., and Ott, M. (2014). Assembly factors monitor sequential hemylation of cytochrome $b$ to regulate mitochondrial translation. J. Cell Biol. 205, 511-524. 
Howden, A.J.M., Geoghegan, V., Katsch, K., Efstathiou, G., Bhushan, B., Boutureira, O., Thomas, B., Trudgian, D.C., Kessler, B.M., Dieterich, D.C., et al. (2013). QuaNCAT: quantitating proteome dynamics in primary cells. Nat. Methods 10, 343-346.

Huang, D.W., Sherman, B.T., and Lempicki, R.A. (2009). Systematic and integrative analysis of large gene lists using DAVID bioinformatics resources. Nat. Protoc. 4, 44-57.

Hünten, S., Kaller, M., Drepper, F., Oeljeklaus, S., Bonfert, T., Erhard, F., Dueck, A., Eichner, N., Friedel, C.C., Meister, G., et al. (2015). p53-Regulated Networks of Protein, mRNA, miRNA, and IncRNA Expression Revealed by Integrated Pulsed Stable Isotope Labeling With Amino Acids in Cell Culture (pSILAC) and Next Generation Sequencing (NGS) Analyses. Mol. Cell. Proteomics 14, 2609-2629.

Imami, K., Milek, M., Bogdanow, B., Yasuda, T., Kastelic, N., Zauber, H., Ishihama, Y., Landthaler, M., and Selbach, M. (2018). Phosphorylation of the Ribosomal Protein RPL12/uL11 Affects Translation during Mitosis. Mol. Cell 72, 84-98.e9.

Isaac, R.S., McShane, E., and Churchman, L.S. (2018). The Multiple Levels of Mitonuclear Coregulation. Annu. Rev. Genet. 52, 511-533.

Ishihama, Y., Rappsilber, J., Andersen, J.S., and Mann, M. (2002). Microcolumns with selfassembled particle frits for proteomics. J. Chromatogr. A 979, 233-239.

Iwasaki, S., and Ingolia, N.T. (2017). The Growing Toolbox for Protein Synthesis Studies. Trends Biochem. Sci. 42, 612-624.

Jardetzky, O. (1963). Studies on the mechanism of action of chloramphenicol. I. The conformation of chlioramphenicol in solution. J. Biol. Chem. 238, 2498-2508.

Klann, K., Tascher, G., and Münch, C. (2020). Functional Translatome Proteomics Reveal Converging and Dose-Dependent Regulation by mTORC1 and elF2a. Mol. Cell 77, 913925.e4.

Lazarou, M., McKenzie, M., Ohtake, A., Thorburn, D.R., and Ryan, M.T. (2007). Analysis of the assembly profiles for mitochondrial- and nuclear-DNA-encoded subunits into complex I. Mol. Cell. Biol. 27, 4228-4237.

Masuda, T., Tomita, M., and Ishihama, Y. (2008). Phase transfer surfactant-aided trypsin digestion for membrane proteome analysis. J. Proteome Res. 7, 731-740.

Masuda, T., Saito, N., Tomita, M., and Ishihama, Y. (2009). Unbiased quantitation of Escherichia coli membrane proteome using phase transfer surfactants. Mol. Cell. Proteomics 8 , 2770-2777.

McShane, E., Sin, C., Zauber, H., Wells, J.N., Donnelly, N., Wang, X., Hou, J., Chen, W., Storchova, Z., Marsh, J.A., et al. (2016). Kinetic Analysis of Protein Stability Reveals AgeDependent Degradation. Cell 167, 803-815.e21.

Moriya, Y., Kawano, S., Okuda, S., Watanabe, Y., Matsumoto, M., Takami, T., Kobayashi, D., Yamanouchi, Y., Araki, N., Yoshizawa, A.C., et al. (2019). The jPOST environment: an integrated proteomics data repository and database. Nucleic Acids Res. 47, D1218-D1224.

Okuda, S., Watanabe, Y., Moriya, Y., Kawano, S., Yamamoto, T., Matsumoto, M., Takami, T., 
Kobayashi, D., Araki, N., Yoshizawa, A.C., et al. (2017). jPOSTrepo: an international standard data repository for proteomes. Nucleic Acids Res. 45, D1107-D1111.

Priesnitz, C., and Becker, T. (2018). Pathways to balance mitochondrial translation and protein import. Genes Dev. 32, 1285-1296.

Protasoni, M., Pérez-Pérez, R., Lobo-Jarne, T., Harbour, M.E., Ding, S., Peñas, A., Diaz, F., Moraes, C.T., Fearnley, I.M., Zeviani, M., et al. (2020). Respiratory supercomplexes act as a platform for complex III-mediated maturation of human mitochondrial complexes I and IV. EMBO J. 39, e102817.

Rappsilber, J., Ishihama, Y., and Mann, M. (2003). Stop and go extraction tips for matrixassisted laser desorption/ionization, nanoelectrospray, and LC/MS sample pretreatment in proteomics. Anal. Chem. 75, 663-670.

Richter-Dennerlein, R., Oeljeklaus, S., Lorenzi, I., Ronsör, C., Bareth, B., Schendzielorz, A.B., Wang, C., Warscheid, B., Rehling, P., and Dennerlein, S. (2016). Mitochondrial Protein Synthesis Adapts to Influx of Nuclear-Encoded Protein. Cell 167, 471-483.e10.

Ritchie, M.E., Phipson, B., Wu, D., Hu, Y., Law, C.W., Shi, W., and Smyth, G.K. (2015). limma powers differential expression analyses for RNA-sequencing and microarray studies. Nucleic Acids Res. 43, e47.

Saladi, S., Boos, F., Poglitsch, M., Meyer, H., Sommer, F., Mühlhaus, T., Schroda, M., Schuldiner, M., Madeo, F., and Herrmann, J.M. (2020). The NADH Dehydrogenase Nde1 Executes Cell Death after Integrating Signals from Metabolism and Proteostasis on the Mitochondrial Surface. Mol. Cell 77, 189-202.e6.

Schanzenbächer, C.T., Sambandan, S., Langer, J.D., and Schuman, E.M. (2016). Nascent Proteome Remodeling following Homeostatic Scaling at Hippocampal Synapses. Neuron 92, 358-371.

Scharfe, C., Lu, H.H.-S., Neuenburg, J.K., Allen, E.A., Li, G.-C., Klopstock, T., Cowan, T.M., Enns, G.M., and Davis, R.W. (2009). Mapping gene associations in human mitochondria using clinical disease phenotypes. PLoS Comput. Biol. 5, e1000374.

Schwanhäusser, B., Gossen, M., Dittmar, G., and Selbach, M. (2009). Global analysis of cellular protein translation by pulsed SILAC. Proteomics 9, 205-209.

Selbach, M., Schwanhäusser, B., Thierfelder, N., Fang, Z., Khanin, R., and Rajewsky, N. (2008). Widespread changes in protein synthesis induced by microRNAs. Nature 455, 58-63.

Signes, A., and Fernandez-Vizarra, E. (2018). Assembly of mammalian oxidative phosphorylation complexes I-V and supercomplexes. Essays Biochem. 62, 255-270.

Stroud, D.A., Surgenor, E.E., Formosa, L.E., Reljic, B., Frazier, A.E., Dibley, M.G., Osellame, L.D., Stait, T., Beilharz, T.H., Thorburn, D.R., et al. (2016). Accessory subunits are integral for assembly and function of human mitochondrial complex I. Nature 538, 123-126.

Taggart, J.C., Zauber, H., Selbach, M., Li, G.-W., and McShane, E. (2020). Keeping the Proportions of Protein Complex Components in Check. Cell Syst 10, 125-132.

Tang, J.X., Thompson, K., Taylor, R.W., and Oláhová, M. (2020). Mitochondrial OXPHOS 
Biogenesis: Co-Regulation of Protein Synthesis, Import, and Assembly Pathways. Int. J. Mol. Sci. 21.

Tong, M., Suttapitugsakul, S., and Wu, R. (2020). Effective Method for Accurate and Sensitive Quantitation of Rapid Changes of Newly Synthesized Proteins. Anal. Chem. 92, 10048-10057.

Topf, U., Uszczynska-Ratajczak, B., and Chacinska, A. (2019). Mitochondrial stress-dependent regulation of cellular protein synthesis. J. Cell Sci. 132.

Uchiyama, J., Ishihama, Y., and Imami, K. (2020). Quantitative nascent proteome profiling by dual pulse labeling with O-propargyl-puromycin and stable isotope labeled amino acids. J. Biochem.

Vidoni, S., Harbour, M.E., Guerrero-Castillo, S., Signes, A., Ding, S., Fearnley, I.M., Taylor, R.W., Tiranti, V., Arnold, S., Fernandez-Vizarra, E., et al. (2017). MR-1S Interacts with PET100 and PET117 in Module-Based Assembly of Human Cytochrome c Oxidase. Cell Rep. 18, 17271738.

Webb, B.D., Diaz, G.A., and Prasun, P. (2020). Mitochondrial translation defects and human disease. JTGG.

Zhang, X., Smits, A.H., van Tilburg, G.B.A., Ovaa, H., Huber, W., and Vermeulen, M. (2018). Proteome-wide identification of ubiquitin interactions using UbIA-MS. Nature Protocols 13, 530550.

\section{Supplemental information}

\section{Contact for reagent and resource sharing}

Further information and requests for reagents may be directed to, and will be fulfilled by the Lead Contact, Koshi Imami (imami.koshi.3z@kyoto-u.ac.jp).

\section{Experimental model and subject details}

\section{Cell culture and pulse labeling}

HEK293T cells obtained from American Type Culture Collection were cultured in Dulbecco's modified eagle medium (DMEM) (Fujifilm Wako, Osaka, Japan) containing 10\% fetal bovine serum (FBS) (Thermo Fisher Scientific, Waltham, USA) in $10 \mathrm{~cm}$ diameter dishes. For pulse SILAC labeling, the cell culture medium was switched to arginine- and lysine-free DMEM (Thermo Fisher Scientific, Waltham, USA) supplemented with 10\% FBS and either "heavy" amino acids [0.398 mM L- $\left({ }^{13} \mathrm{C}_{6},{ }^{15} \mathrm{~N}_{4}\right)$-arginine (Arg"10") and $0.798 \mathrm{mM} \mathrm{L-}\left({ }^{13} \mathrm{C}_{6},{ }^{15} \mathrm{~N}_{2}\right)$-lysine (Lys"8")] or "medium-heavy" amino acids [0.398 mM L- $\left({ }^{13} \mathrm{C}_{6}\right)$-arginine (Arg"6") and $0.798 \mathrm{mM} \mathrm{L-}$ 
$\left(\mathrm{D}_{4}\right)$-lysine (Lys"4")] (Cambridge Isotope Laboratories, Tewksbury, USA). For chloramphenicol (CAP) (Fujifilm Wako) treatment, HEK293T cells were first pre-incubated with the corresponding SILAC medium for $8 \mathrm{hr}$, and then further incubated for $16 \mathrm{hr}$ in the presence of $10 \mu \mathrm{g} / \mathrm{mL}$ CAP or vehicle (DMSO). The cells were washed and harvested in ice-cold PBS, and pelleted by centrifugation at $600 \times g$ for $5 \mathrm{~min}$ at $4^{\circ} \mathrm{C}$. Label-swap (biological duplicate) experiments were performed. All cells were maintained in a humidified $37^{\circ} \mathrm{C}$ incubator with $5 \% \mathrm{CO}_{2}$.

\section{Methods details}

\section{Mitochondria isolation}

Mitochondria were isolated according to (Frezza et al., 2007). For pSILAC samples, corresponding medium-heavy and heavy-labeled cells were combined at this stage. The cell pellets were resuspended in $500 \mu \mathrm{L}$ of ice-cold mitochondria isolation buffer (10 mM Tris-MOPS [3-(N-morpholino)propanesulfonic acid] $\mathrm{pH} 7.4$ containing $1 \mathrm{mM}$ EGTA/Tris and $200 \mathrm{mM}$ sucrose). The cells were homogenized using a glass/Teflon Potter Elvehjem homogenizer (2 mL volume; 40 strokes). The homogenate was transferred to a new $1.5 \mathrm{~mL}$ tube and centrifuged at $600 \times \mathrm{g}$ for $10 \mathrm{~min}$ at $4^{\circ} \mathrm{C}$. The supernatant was transferred to a new $1.5 \mathrm{~mL}$ tube and centrifuged at $7,000 \times \mathrm{g}$ for $10 \mathrm{~min}$ at $4^{\circ} \mathrm{C}$. The pellet containing mitochondria was resuspended in $100 \mu \mathrm{L}$ of ice-cold mitochondria isolation buffer and centrifuged at $7,000 \times \mathrm{g}$ for $10 \mathrm{~min}$ at $4^{\circ} \mathrm{C}$. The supernatant was discarded, and the pellet was used as the mitochondrial fraction.

\section{Protein digestion}

Protein digestion was performed according to the phase-transfer surfactant (PTS)-aided digestion protocol, as described previously (Masuda et al., 2008, 2009). Briefly, the mitochondrial fraction was lysed with PTS buffer [12 mM sodium deoxycholate (SDC) (Fujifilm Wako), $12 \mathrm{mM}$ sodium $\mathrm{N}$-lauroyl sarcosinate (SLS) (Fujifilm Wako) in $0.1 \mathrm{M}$ Tris- $\mathrm{HCl} \mathrm{pH}$ 8.0] and incubated with $10 \mathrm{mM}$ DTT at $37^{\circ} \mathrm{C}$ for $30 \mathrm{~min}$, followed by alkylation with $50 \mathrm{mM}$ iodoacetamide at $37^{\circ} \mathrm{C}$ for $30 \mathrm{~min}$ in the dark. The samples were diluted 5 times with $50 \mathrm{mM}$ ammonium bicarbonate. To optimize the digestion protocol for mitochondrial translation products, proteins were digested with 1) chymotrypsin (Promega, Madison, USA), 2) chymotrypsin and lysyl endopeptidase (lys-C) (Fujifilm Wako) 3) lys-C and trypsin (Promega) and 4) chymotrypsin and trypsin at a protein-to-protease ratio of $50: 1(\mathrm{w} / \mathrm{w})$ overnight at $37^{\circ} \mathrm{C}$ on a shaking incubator. For the pSILAC experiments, proteins were first digested with lys-C for $3 \mathrm{hr}$ at $37^{\circ} \mathrm{C}$ and then with trypsin overnight at $37^{\circ} \mathrm{C}$ on a shaking incubator. Next day, an equal 
volume of ethyl acetate (Fujifilm Wako) was added to the sample and digestion was quenched by adding $0.5 \%$ TFA (final concentration). The samples were shaken for $1 \mathrm{~min}$ and centrifuged at $16,000 \times \mathrm{g}$ for $2 \mathrm{~min}$ at $25^{\circ} \mathrm{C}$. The organic phase containing SDC and SLS was discarded. The resulting peptide solution was evaporated in a SpeedVac and the residue was resuspended in $200 \mu \mathrm{L} 0.1 \%$ TFA 5\% acetonitrile (ACN). The peptides were desalted with an SDB-XC StageTip (Rappsilber et al., 2003) or fractionated into 7 fractions using an SDB-XC-SCX StageTip (Adachi et al., 2016). The sample solution was evaporated in a SpeedVac and the residue was resuspended in $0.5 \%$ TFA and $4 \%$ ACN.

\section{LC/MS/MS analysis}

Nano-scale reversed-phase liquid chromatography coupled with tandem mass spectrometry (nanoLC/MS/MS) was performed on an Orbitrap Fusion Lumos mass spectrometer (Thermo Fisher Scientific), connected to a Thermo Ultimate 3000 RSLCnano pump and an HTC-PAL autosampler (CTC Analytics, Zwingen, Switzerland) equipped with a self-pulled analytical column (150 mm length × $100 \mu \mathrm{m}$ i.d.) (Ishihama et al., 2002) packed with ReproSil-Pur C18AQ materials ( $3 \mu \mathrm{m}$, Dr. Maisch $\mathrm{GmbH}$, Ammerbuch, Germany). The mobile phases consisted of (A) $0.5 \%$ acetic acid and (B) $0.5 \%$ acetic acid and $80 \%$ ACN. For pSILAC experiments, peptides were eluted from the analytical column at a flow rate of $500 \mathrm{~nL} / \mathrm{min}$ by altering the gradient: $5-10 \%$ B in $5 \mathrm{~min}, 10-40 \%$ B in $60 \mathrm{~min}, 40-99 \%$ B in $5 \mathrm{~min}$ and $99 \%$ for $5 \mathrm{~min}$, and 300 min gradient was used for the biochemical optimization. The Orbitrap Fusion Lumos instrument was operated in the data-dependent mode with a full scan in the Orbitrap followed by MS/MS scans for $3 \mathrm{sec}$ using higher-energy collisional dissociation (HCD). The applied voltage for ionization was $2.4 \mathrm{kV}$. The full scans were performed with a resolution of 120,000 , a target value of $4 \times 10^{5}$ ions, and a maximum injection time of $50 \mathrm{~ms}$. The MS scan range was $\mathrm{m} / \mathrm{z} 300-1,500$. The MS/MS scans were performed with 15,000 resolution, $5 \times 10^{4}$ target value, and $50 \mathrm{~ms}$ maximum injection time. The isolation window was set to 1.6, and the normalized HCD collision energy was 30. Dynamic exclusion was applied for $20 \mathrm{sec}$.

\section{Database searching and protein quantification}

All raw files were analyzed and processed by MaxQuant (v1.6.0.13) (Cox and Mann, 2008). Search parameters included two missed cleavage sites and variable modifications such as methionine oxidation, protein N-terminal acetylation, and SILAC-specific modifications [L$\left({ }^{13} \mathrm{C}_{6},{ }^{15} \mathrm{~N}_{4}\right)$-arginine, $\quad \mathrm{L}-\left({ }^{13} \mathrm{C}_{6},{ }^{15} \mathrm{~N}_{2}\right)$-lysine, $\quad \mathrm{L}-\left({ }^{13} \mathrm{C}_{6}\right)$-arginine, $\quad \mathrm{L}-\left(\mathrm{D}_{4}\right)$-lysine $] \quad$ Cysteine carbamidomethylation was set as a fixed modification. The peptide mass tolerance was $4.5 \mathrm{ppm}$, 
and the MS/MS tolerance was $20 \mathrm{ppm}$. The database search was performed with Andromeda (Cox et al., 2011) against the UniProt/Swiss-Prot human database (downloaded on 2014-06) with common serum contaminants and enzyme sequences. The false discovery rate (FDR) was set to $1 \%$ at the peptide spectrum match (PSM) level and protein level. The 'match between runs' functions were employed. For protein quantification, a minimum of one unique peptide ion was used, and to ensure accurate quantification, we required proteins to be quantified in all samples for further analysis. Protein intensities from SILAC medium-heavy and heavy channels were normalized using the default settings of the R package of DEP (Zhang et al., 2018) to correct for mixing error between the two SILAC labeled lysates. P-values were computed based on differential expression of proteins using protein-wise linear models and empirical Bayes statistics through the limma function (Ritchie et al., 2015).

\section{Assessment of coverage of mitochondrial translation products and measurement time (related to Figure 1)}

There are a number of studies on cellular translation using PSILAC, AHA and/or puromycin, as described in the introduction. To compare our findings with those of previous reports, we chose studies that had employed pSILAC with medium-heavy and heavy amino acids (i.e., triplex SILAC) and that showed reasonable high proteome coverage. Recent studies using AHA, puromycin (and its analog) or dynamic SILAC-tandem-mass tag (TMT) were also included. If multiple experiments were performed within a study, one specific experiment with the highest proteome coverage was chosen (see Figure 1). The measurement time per experiment is a conservative estimate, as some studies provided only the LC gradient time and did not mention total measurement time.

\section{Gene ontology (GO) enrichment analysis (related to Figure 2A)}

GO enrichment analysis was performed using DAVID (Huang et al., 2009). To identify enriched GO terms in the mitochondrial fraction and total cell lysate, we used only proteins quantified in all of the digestion protocols. The top 3 enriched terms for cellular components are shown. FDRs were corrected by the Benjamini-Hochberg method.

\section{Data and software availability}

The proteomics data have been deposited to the ProteomeXchange Consortium via jPOST (Moriya et al., 2019; Okuda et al., 2017) partner repository with the dataset identifier JPST001007 (PXD022476 for ProteomeXchange). 


\section{Quantification and statistical analysis}

The type of statistical test (e.g., t-test using protein-wise linear models and empirical Bayes statistics) is annotated in the Figure legend and/or in the Methods and Resources segment specific to the analysis. In addition, statistical parameters such as the value of $n$, mean/median and significance level are reported in the Figures and/or in the Figure Legends. Statistical analyses were performed using $\mathrm{R}$ as described in Methods and Resources for each individual analysis. 
Fig. 1

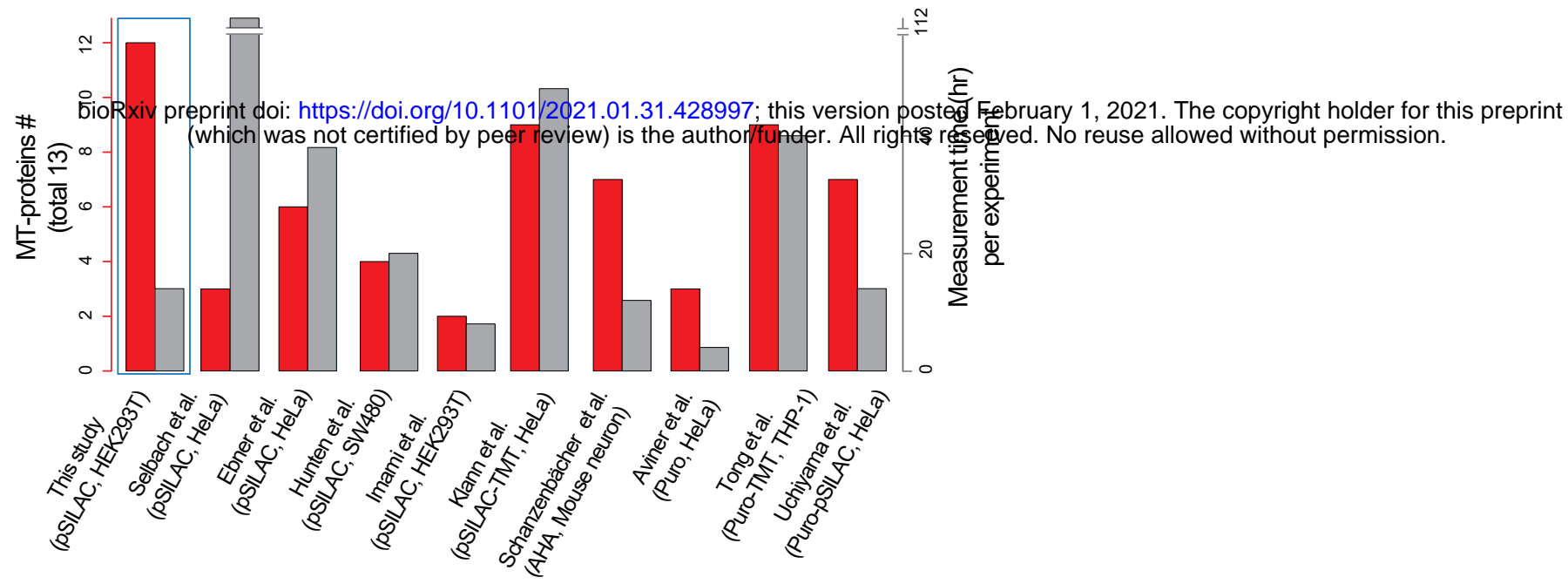

Figure 1: Comparison of our method with previous studies.

The coverage of the MT-proteins (left axis; red color) and the total LC/MS/MS measurement time (right axis; grey color) are shown. Methods and cell types are shown in parentheses. The present study gave the highest coverage of MT-proteins (12 proteins) with the shortest measurement time (14 hr). 
Fig. 2
A
$\mathrm{B}$
C 0
Peptide \# $>8$

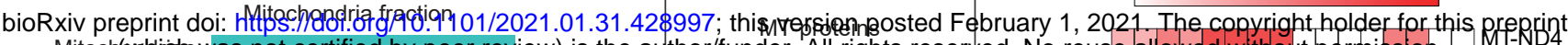
Mitoch(omblich was not certified by peer review) is the author/funder. All rights reserved. No reuse allowed without permission. Membrane
Mitochondrial inner membrane
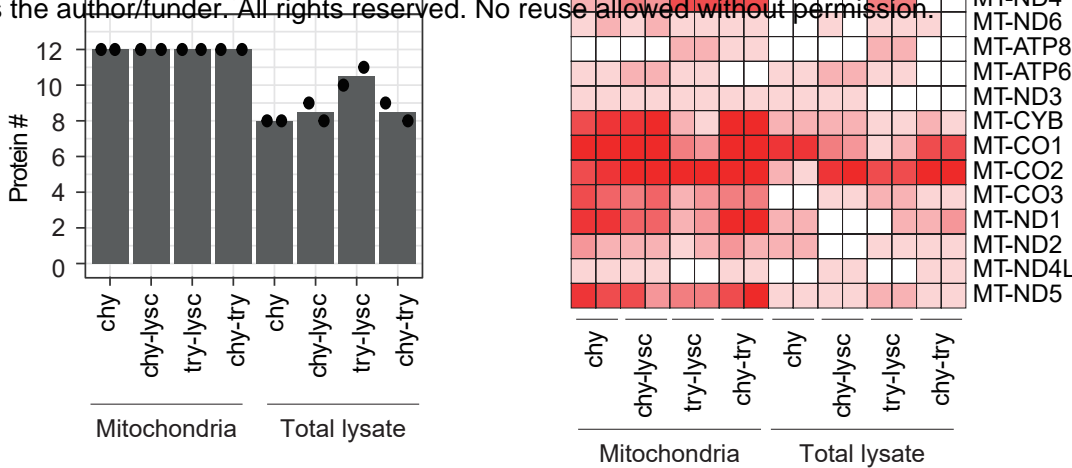

Figure 2: Optimization of biochemical conditions.

(A) The top3 GO terms enriched in the mitochondrial fraction (top: light green) and total cell lysate (bottom: grey). (B) Coverage of the MT-proteins obtained under 8 different conditions. The bars show the average number of identified proteins from two independent experiments (filled-circle). (C) A heatmap showing the number of identified peptides per MT-protein. 
Fig. 3

A

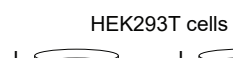

B

MT-CO3

MT-proteins

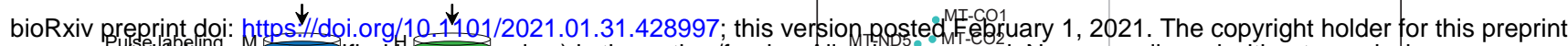
(which was not certified by peer review) is the author/funder. All rights respr-Kegd. No reuse allowed without permission.
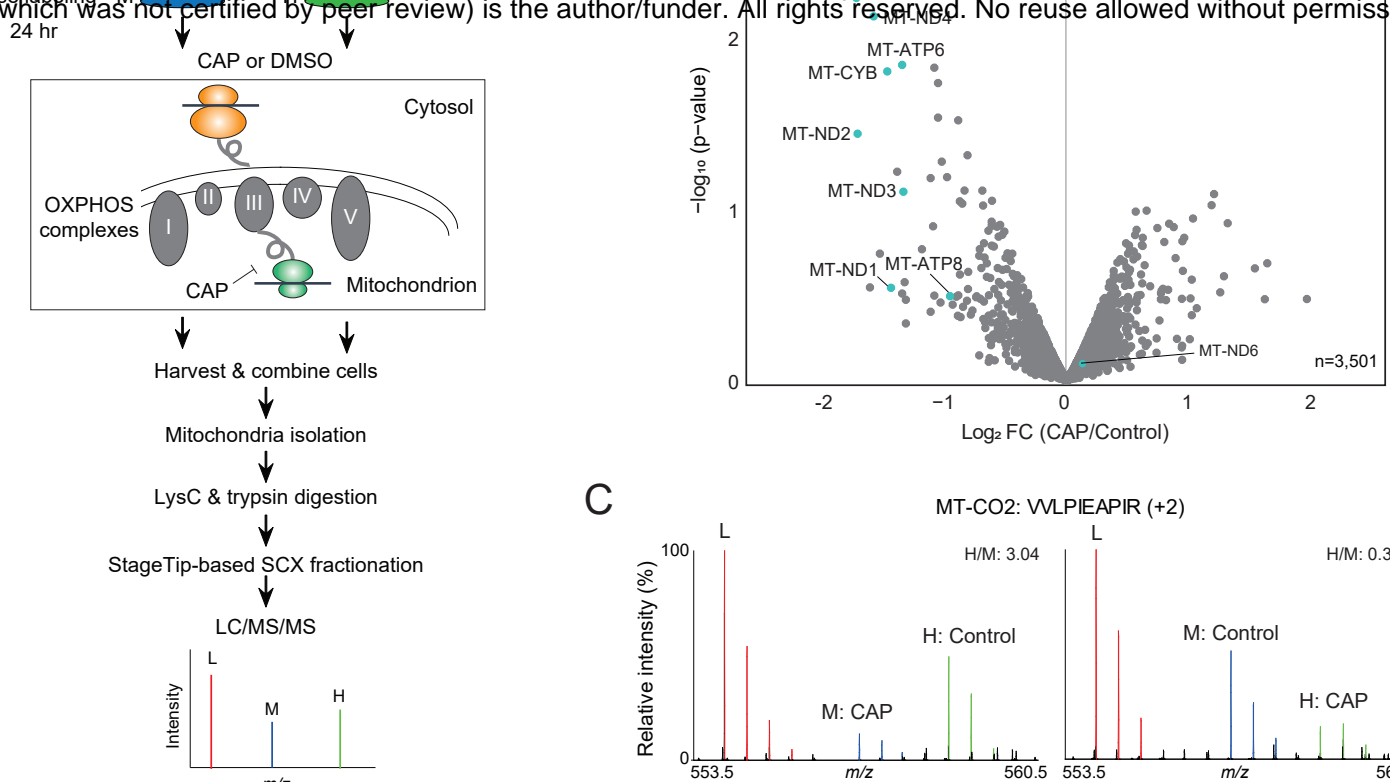

C

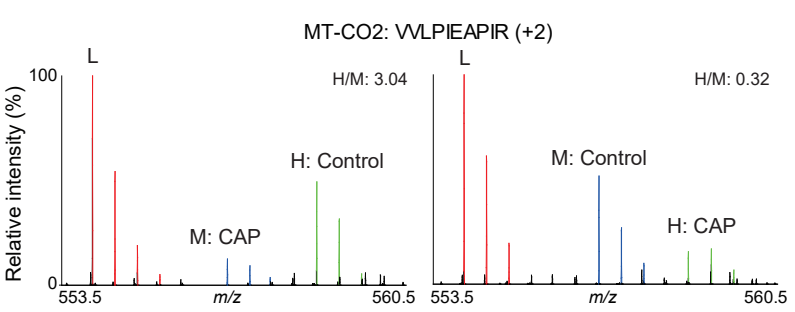

Figure 3: pSILAC experiments.

(A) Experimental scheme of pSILAC. HEK293T cells were pulsed-labeled with medium-heavy or heavy amino acids in the presence of CAP or DMSO. Label-swap experiments were performed. (B) A volcano plot showing log2 fold-change (CAP/DMSO control) and -log10 p-value. The MT-proteins are indicated by light green filled circles. (C) MS spectrum of an MT-CO2 peptide (VVLPIEAPIR, +2), as an example. 
Fig. 4

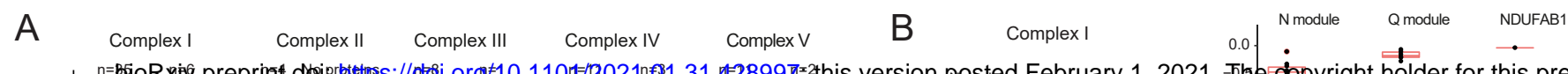

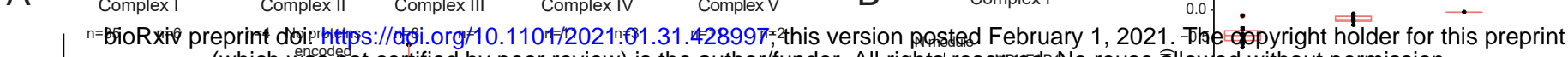
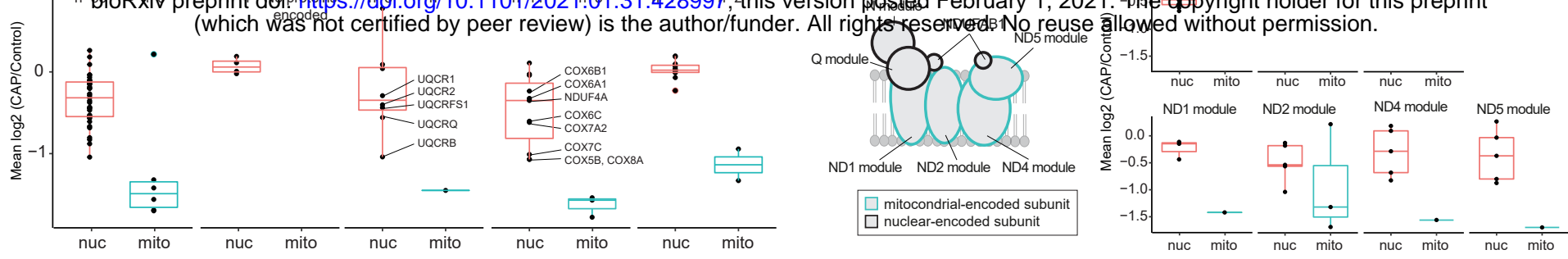

C

Complex IV
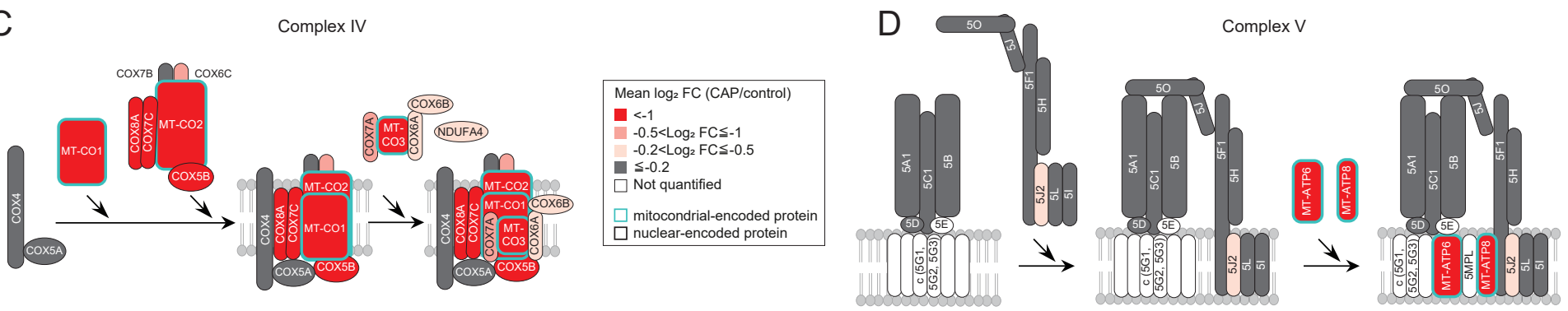

$\mathrm{E}$

E Complex III

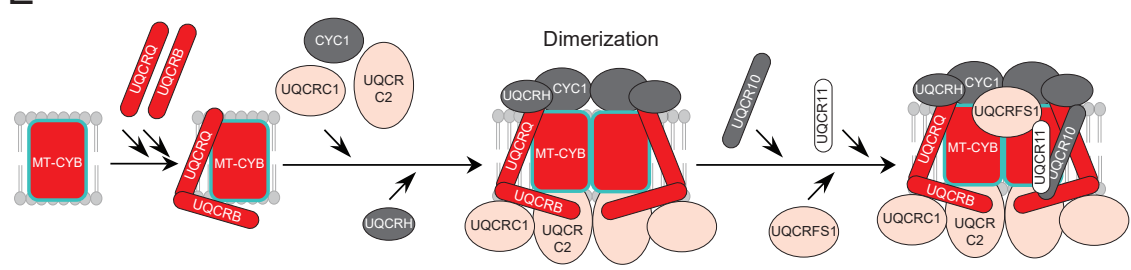

UQCRQ: HVISYSLSPFEQR (+2)

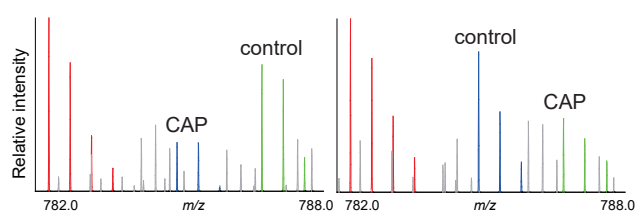

CYC1: GLLSSLDHTSIR (+2)
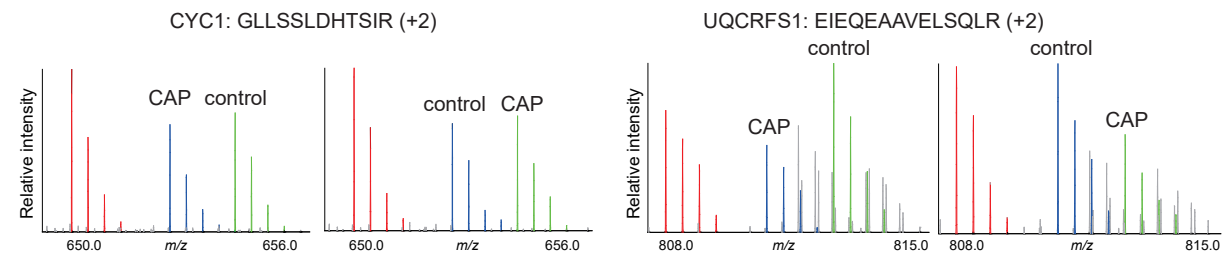

Figure 4: (Post-)translational control and OXPHOS complex assembly.

(A) Box plots showing log2 fold-change (CAP/Control) of nuclear- ( "nuc") and mitochondrial-encoded proteins ( "mito" ) in individual OXPHOS complexes. (B) Positions of subunits in complex I (NADH:ubiquinone oxidoreductase) based on humans (Zhu et al., 2016) (right) and boxplots showing log2 fold-change (CAP/Control) for "nuc" and "mito" proteins. (C) An assembly model for complex IV (or cytochrome c oxidase) based on humans (Vidoni et al., 2017). Quantitative values (i.e., log2 FCs (CAP/Control)) are indicated by color. (D) An assembly model for complex V (or ATP synthase) based on humans (He et al., 2018). (E) A reported assembly model for complex III (cytochrome bc1 complex) based on yeast (Fernández-Vizarra and Zeviani, 2015) (top) and exemplary MS spectra for the nuclear-encoded proteins (UQCRQ, CYC1 and UQCRFS1) derived peptides (bottom). 